\title{
EQUILIBRIUM SHAPE AND INTERFACE ROUGHENING OF SMALL LIQUID Pb INCLUSIONS IN SOLID AI
}

\author{
H. Gabrisch, L. Kjeldgaard ${ }^{1}$, E. Johnson ${ }^{1}$ and U. Dahmen \\ National Center for Electron Microscopy, Lawrence Berkeley National Laboratory \\ 1 Cyclotron Road, Berkeley, California 94720, USA \\ Niels Bohr Institute, Ørsted Laboratory, University of Copenhagen, Denmark
}

\begin{abstract}
The shape of liquid $\mathrm{Pb}$ inclusions embedded in a solid Al matrix was investigated at temperatures between 300 and $500^{\circ} \mathrm{C}$ using in-situ electron microscopy. Inclusion shapes in the size range from a few nanometers to about 150 nanometers were found to depend on size, temperature and thermal history. During isothermal annealing after melting, small inclusions rounded off while larger inclusions remained faceted until the temperature was raised to about $500^{\circ} \mathrm{C}$. During subsequent cooling, inclusions refaceted, although less strongly than during heating. The shape hysteresis between heating and cooling cycles was found to be due to the barrier of ledge nucleation necessary to advance the faceted interfaces. It is shown that this kinetic barrier can explain the observed dependence on size and temperature, and that the $\{111\}$ interface undergoes a roughening transition at about $550^{\circ} \mathrm{C}$. Even under conditions of kinetic limitation it was possible to measure local equilibrium by modeling kinetically limited inclusions as a droplet in a crevice. For this type of measurement, the hysteresis between heating and cooling cycles disappeared, and the true equilibrium shape could be derived. The anisotropy of interfacial energy was shown to be significantly smaller than previously reported, and at about $2 \%$, similar to the anisotropy of the surface energy for fcc metals. From the width of facets on the equilibrium shape, the step energy was determined to be $\varepsilon=1.9 \cdot 10^{-11} \mathrm{~J} / \mathrm{m}$ at $350^{\circ} \mathrm{C}$.
\end{abstract}


Keywords: interface, phase transformation, aluminium alloy, equilibrium shape, transmission electron microscopy 


\section{INTRODUCTION}

The thermal behavior of $\mathrm{Pb}$ inclusions in a solid $\mathrm{Al}$ matrix has been investigated by a number of groups for the last three decades because $\mathrm{Al}-\mathrm{Pb}$ is a good model alloy for the study of solid-liquid equilibrium shapes. The phase diagram is a simple monotectic with a miscibility gap in the liquid state, negligible solubility in the solid state and no intermediate phases (see Figure 1). Both, $\mathrm{Al}$ and $\mathrm{Pb}$ are face-centered cubic with a $22 \%$ difference in lattice parameter $\left(\mathrm{a}_{\mathrm{Al}}=0.405 \mathrm{~nm}, \mathrm{a}_{\mathrm{Pb}}=0.495 \mathrm{~nm}\right)$. $\mathrm{Al}$ and $\mathrm{Pb}$ have widely separated melting points (660 and $327^{\circ} \mathrm{C}$, respectively), and volume strains are easily accommodated at temperatures above $200^{\circ} \mathrm{C}$ when thermal vacancies become mobile in Al. The present investigation is concerned with liquid $\mathrm{Pb}$ inclusions in solid $\mathrm{Al}$. However, for reference, previous work on both solid and liquid $\mathrm{Pb}$ inclusions in $\mathrm{Al}$ will be reviewed briefly.

Solid $\mathrm{Pb}$ inclusions in $\mathrm{Al}$ have been found to adopt a cube-cube orientation relationship and form defect-free precipitates in the shape of a cuboctahedron - an octahedron with eight $\{111\}$ facets truncated by six $\{100\}$ facets [1]. Herring has shown that at equilibrium, the ratio of distances between the $\{100\}$ and $\{111\}$ facets is a measure of their interfacial energy ratio $\gamma_{\{100\}} / \gamma\{111\}$ [2]. Since all inclusions are parallel to the matrix lattice but isolated from each other by the limited solid solubility, the Al matrix acts as an alignment field and an isolating medium. In the nanoscale size regime, solid $\mathrm{Pb}$ inclusions display magic size behavior [3], and their equilibrium shape is affected by the edge energy of the faceted shapes [4]. Solid inclusions at grain boundaries were found to adopt compound shapes that depend on the crystallographic characteristics of the grain boundary [5]. 
Liquid $\mathrm{Pb}$ inclusions in solid $\mathrm{Al}$ were first investigated by Thackery and Nelson [6] who measured particle shapes after ion-implantation and subsequent cooling from their liquid state at $400^{\circ} \mathrm{C}\left(\mathrm{T}_{\mathrm{m}}=327^{\circ} \mathrm{C}\right)$. The particles were a few hundred $\mathrm{nm}$ in diameter and contained both $\{111\}$ and $\{100\}$ facets. The authors believed that the inclusion shape measured after cooling to room temperature was in equilibrium, and that the aspect ratio of $\gamma_{100} / \gamma_{111}$ of 1.11 was a measure of the interface energy anisotropy at $400^{\circ} \mathrm{C}$.

Subsequently McLean used X-ray radiography to study the kinetics of spheroidization of long cylinders or rods of liquid $\mathrm{Pb}$, several $\mu \mathrm{m}$ in diameter [7]. The break-up of the rods was found to be controlled by the diffusion of $\mathrm{Al}$ through liquid $\mathrm{Pb}$. Somewhat smaller inclusions were examined later by McLean and Loveday using high voltage electron microscopy with a hot stage for in-situ observation at about $600^{\circ} \mathrm{C}$ [8]. It was found that inclusions with aspect ratios larger than about 8 broke into several spheres whereas inclusions with a lower aspect ratio tended to spheroidize under capillary forces. Spherical inclusions larger than about $0.12 \mu \mathrm{m}$ in diameter migrated in a temperature gradient while smaller particles were immobile. Equilibrated particles tended to be spherical without apparent facets, and even during thermal migration, the inclusions remained spherical. From measurements of dihedral angles of the liquid with grain boundaries, an anisotropy of the solid-liquid interfacial energy as low as 3-4\% was suggested.

To resolve the discrepancy between the observations of Thackery and Nelson [6] and McLean [7], McCormick et al. [9] compared $\mathrm{Pb}$ inclusions quenched from 610 and $410^{\circ} \mathrm{C}$ by ex-situ transmission electron microscopy. Their results confirmed both observations in that particles smaller than about $0.4 \mu \mathrm{m}$ (like those of Thackery and Nelson) were faceted whereas larger particles (like those of McLean) were more rounded. The authors interpreted this as a possible size dependence of the shape, but 
commented that the equilibrium shape remained unknown, possibly due to the influence of dislocations on particle shapes.

Employing the reverse geometry in the $\mathrm{Ag}-\mathrm{Pb}$ system (solid $\mathrm{Ag}$ particles in a liquid $\mathrm{Pb}$ matrix rather than liquid $\mathrm{Pb}$ particles in a solid $\mathrm{Ag}$ matrix) to study the same solid-liquid interface in the similar system of Ag-Pb, Camel et al. [10] measured the shapes of large solid $\mathrm{Al}$ inclusions in a liquid $\mathrm{Ag}-\mathrm{Pb}$ alloy quenched from near the monotectic temperature, where solid $\mathrm{Ag}$ is in equilibrium with a $\mathrm{Pb}$-rich melt (equivalent to Figure 1). Using scanning electron microscopy, these authors measured the aspect ratio of $\mathrm{Ag}$ crystals, etched from the $\mathrm{Pb}$-rich matrix after quenching. The anisotropy of the interfacial energy between solid $\mathrm{Ag}$ and liquid $\mathrm{Pb}$ was found to be of the same magnitude (a few percent) as a typical solid-vapor interface.

By contrast, Moore et al. found a much larger degree of anisotropy by studying liquid $\mathrm{Pb}$ inclusions in $\mathrm{Al}$ in-situ during heating in a transmission electron microscope [1,11]. Direct observations of the melting of individual particles about $0.2 \mu \mathrm{m}$ in size showed that after melting, the liquid particles maintained extended $\{111\}$ facets. From the shapes of such particles, these authors found an anisotropy of $14 \%$ just above the melting point of $\mathrm{Pb}\left(327^{\circ} \mathrm{C}\right)$, decreasing to zero at about $550^{\circ} \mathrm{C}$. Although roughly consistent with the report by Camel et al. at high temperature, these observations indicated a large increase of anisotropy at lower temperature.

Size-dependent thermal behavior of $\mathrm{Pb}$ inclusions in $\mathrm{Al}$ was first reported by Johnson et al. who measured superheating of about $60 \mathrm{~K}$ [12] and even higher [13] for particles about $14 \mathrm{~nm}$ in size in alloys made by ion implantation. Similar behavior with superheating up to $40 \mathrm{~K}$ was also found by Zhang and Cantor in material with somewhat larger particles made by melt spinning [14]. Using X-ray diffraction during heating, Gråbæk et al.[15] observed the disappearance of $\{100\}$ truncation rods at $608 \mathrm{~K}$, while the inclusions were 
still solid. This was interpreted as evidence for a roughening of the $\{100\}$ facets before the onset of melting.

Upon cooling from the liquid state, inclusions solidify with an undercooling of about 30K. The precise degree of undercooling also displays a dependence on temperature, though less so than the superheating during melting. The nucleation kinetics of $\mathrm{Pb}$ inclusions in $\mathrm{Al}$ and $\mathrm{Cu}$ matrices has been investigated in detail by Cantor and coworkers $[11,16,17]$ and related to the contact angle of solid $\mathrm{Pb}$ during heterogeneous nucleation from the liquid.

In the present study, we investigate the shape of liquid $\mathrm{Pb}$ inclusions in $\mathrm{Al}$ over a range of sizes and temperatures by in-situ transmission electron microscopy. Previous observations showed a strong dependence of particle shapes on size, temperature and thermal history $[18,19]$. As will be shown below, these observations can be explained in terms of a kinetic limitation that can prevent faceted particles from reaching their equilibrium shape below the roughening transition temperature of the $\{111\}$ interface facets [20]. After investigating the kinetic barrier that can limit the approach to global equilibrium, the present study measures local equilibrium. The analysis presented here is able to reconcile the discrepancies between all previous observations.

\section{METHODS AND MATERIALS}

Ribbons of Al $99.999 \%$ containing 0.5 at-\% of $\mathrm{Pb} 99.9999$ were produced by rapid solidification. The specimens were annealed at $280^{\circ} \mathrm{C}$ for 2 hours in an Ar atmosphere to equilibrate the solid inclusions and anneal out defects. TEM foils were prepared by punching 3-mm discs from the ribbons followed by twin jet thinning using an electrolyte of $25 \% \mathrm{HNO}_{3}$ in methanol at $-40^{\circ} \mathrm{C}$ and $55 \mathrm{~mA}$. In-situ observations were made in a JEOL 200CX microscope using a water-cooled Gatan double tilt heating holder. Grains with an initial orientation close to $<110>$ were aligned parallel to the $<110>$ zone axis, 
and the foil was heated to $360^{\circ} \mathrm{C}, 420^{\circ} \mathrm{C}, 460^{\circ} \mathrm{C}, 490^{\circ} \mathrm{C}$ and $505^{\circ} \mathrm{C}$. At each temperature the specimen was held for 2-3 minutes before recording a micrograph. After equilibrating above $500^{\circ} \mathrm{C}$ the shape of the same particle was recorded during cooling. Extended heating above $500^{\circ} \mathrm{C}$ was not routinely possible in foils thin enough to be electron transparent at $200 \mathrm{kV}$ because above this temperature particles were lost to the surfaces before reaching equilibrium. The orientation of the matrix was monitored using the diffraction pattern and corrected when necessary throughout the experiment. Every foil was used for one melting-solidification experiment only. The temperature reading was verified after the end of the experiment by observing the melting point of unconstrained lead assembled at the edge of the foil during re-heating. In a dark field image, the contrast disappears when the lead melts. Using this technique, the accuracy of the temperature reading was found to be better than $\pm 10^{\circ} \mathrm{C}$. At equilibrium, the aspect ratio between the spherical caps that replace the $\{100\}$ facets upon melting and the $\{111\}$ interface facets can be taken as a measure of the anisotropy of the interfacial energy between solid $\mathrm{Al}$ and liquid $\mathrm{Pb}$ [1]. Micrographs recorded in $<110>$ zone axis orientation were digitized for shape measurement. The aspect ratios of distances between spherical caps replacing the $\{100\}$ facets and the remaining $\{111\}$ facets were measured manually or by a computer program. Measurement of local equilibrium was made on digitized images using an automated 7-parameter least squares fit of a model to the observed shapes [21].

\section{RESULTS}

\section{Shape dependence on size}

In-situ observations during heating above the bulk melting point of $\mathrm{Pb}\left(327^{\circ} \mathrm{C}\right)$ confirmed the earlier reports of a size-dependent superheating [12]. Figure 2a, recorded at $423^{\circ} \mathrm{C}$, shows a typical array of inclusions in a bright field micrograph near a $<110>$ 
zone axis orientation. In this orientation, the cuboctahedral shape of solid particles projects as a rhombus truncated by $<100>$ facets. Upon melting, the $<100>$ truncating facets give way to rounded caps, and the moiré fringes disappear. Note that in Figure 2a the smallest particle (arrowed) is still solid, demonstrating directly that superheating can reach $96 \mathrm{~K}$ for inclusions $5 \mathrm{~nm}$ in size. All other inclusions are liquid. From this micrograph it is apparent that the liquid inclusion shape at a given temperature depends on size. While the smallest liquid particles $(<10 \mathrm{~nm})$ appear almost perfectly round, the larger liquid particles are faceted. The largest inclusion is about $60 \mathrm{~nm}$ in size and exhibits clear $\{111\}$ facets joined by rounded caps. It is evident that the degree of faceting increases with particle size. The simplest measure of the particle shape is the aspect ratio, given by the distance between the rounded caps over the distance between flat $\{111\}$ facets. A plot of the aspect ratio as a function of size at a constant temperature of $\mathrm{T}=427^{\circ} \mathrm{C}$ is given in Figure $2 \mathrm{~b}$. Even for the limited range of inclusion sizes available in the melt-quenched material, the size-dependence of the shape is clearly visible.

It was found that during continued heating, such particles maintained a remarkably stable shape until they suddenly rounded off at a critical temperature $T_{r}$, which again depended on size. However, this shape change was not fully reversed during subsequent cooling.

\section{Shape dependence on temperature}

The development of the particle shape through a full heating and cooling cycle is illustrated in Figure 3. This sequence of micrographs shows how the shape changes with temperature during heating and cooling. After melting at around $350-360^{\circ} \mathrm{C}$ the $\{100\}$ facets round off while $\{111\}$ facets remain flat. With increasing temperature the amount of faceting on $\{111\}$ surface planes recedes marginally until in the range of $460^{\circ} \mathrm{C}$ considerable particle rounding sets in. At $507^{\circ} \mathrm{C}$ the particles are almost spherical. During cooling the $\{111\}$ faces reappear to some extent, but the anisotropy of the particle 
shape at $391{ }^{\circ} \mathrm{C}$ and $366^{\circ} \mathrm{C}$ is much smaller than at the same temperatures during heating. To highlight this hysteresis, two micrographs taken at the same temperature during heating and cooling are outlined by frames ( $b$ and $h$ ). Since the equilibrium shape is unique, at least one of the two shapes observed is not at equilibrium. Upon melting, the change in shape from the fully faceted solid state to the partially rounded liquid state occurs very rapidly (within less than a second). It therefore appears unlikely that the approach to equilibrium at a higher temperature, where atomic mobility is significantly higher, is kinetically limited. Nevertheless, experiments were conducted to test the longterm stability of the two different shapes that are found at the same temperature during heating and cooling. Figure 4 shows the shape of particles at $450^{\circ} \mathrm{C}$; immediately after reaching the annealing temperature of $450^{\circ} \mathrm{C}$ during heating (a), and after holding for $9 \mathrm{~h}$ at this temperature (b), showing no apparent change in shape. For comparison, a similar pair of particles after a brief temperature excursion to $527^{\circ} \mathrm{C}$ followed by cooling to $450^{\circ} \mathrm{C}$ is shown in (c) and again their shape is unaltered after holding for $9 \mathrm{~h}$ at $450^{\circ} \mathrm{C}(\mathrm{d})$. The two sets of shapes during heating and cooling are clearly different, and this experiment demonstrates that the shape hysteresis at this temperature is stable over extended periods of time.

Figure 5 shows a quantitative determination of the shape evolution during thermal cycling. As in Figure $2 b$, the shape is measured as the aspect ratio of distances between pairs of rounded $\{100\}$ segments and pairs of $\{111\}$ facets. To capture the size dependence of particle rounding the data was divided into three size groups for particle diameters $50-75 \mathrm{~nm}, 75-100 \mathrm{~nm}$ and 100-150nm. In each group the development of shape starts with a high aspect ratio above 1.2 immediately after melting at around $360^{\circ} \mathrm{C}$ and decreases to an aspect ratio below 1.1 above $500^{\circ} \mathrm{C}$. During subsequent cooling a small but steady increase of aspect ratio is observed. The comparison of the graphs for the three size groups clearly shows that the smaller particles in the $50-75 \mathrm{~nm}$ category have a 
smaller hysteresis and round off at a lower temperature than the larger particles with a diameter between $100-150 \mathrm{~nm}$. This is in agreement with the static observations in Figure 2.

\section{Kinetic limitation}

The effect of size was measured systematically, and a plot of observed rounding temperature $T_{r}$ versus particle sizes is presented in Figure 6. The dependence of $T_{r}$ on size is nonlinear, with a strong increase in the small size regime and a much more gradual increase in the large size regime. The data clusters represent the bimodal size distribution typical for melt-spun monotectic alloys [1]. Assuming the existence of an energy barrier that needs to be overcome before particles attain their equilibrium shape the following model can be derived:

Comparing the rounded particle shape to the faceted shape, one finds that the $\{111\}$ interface needs to be moved into the aluminum matrix to approach the spherical equilibrium shape. This is illustrated with the superposition of a particle shape before and after rounding in Figure 7a. Because the $\{111\}$ interface is faceted, it must advance by a ledge mechanism which is controlled by the nucleation of a stable two-dimensional island on the facet. The need for removal of $\{111\}$ Al layers for island nucleation is shown schematically in Figure 7b. Note that in the reverse direction a kinetic limitation is not expected because attachment sites are readily available at the curved interface.

The change in Gibbs free energy associated with nucleation of an island of radius $r$ and height $h$ is

$$
\Delta G(r)=2 \pi r \varepsilon-\pi r^{2} h \Delta G_{v o l}
$$


where $\varepsilon$ is the step energy and $\Delta \mathrm{G}_{\mathrm{vol}}$ the change in volume free energy of the particle. The minimum size at which the nucleated island will be stable $(\partial \Delta \mathrm{G} / \partial \mathrm{r}=0)$ corresponds to an activation barrier

$$
\Delta G^{*}=\frac{\pi \varepsilon^{2}}{h \Delta G_{v o l}} .
$$

The frequency $\mathrm{f}$ of nucleation of a stable island is then given by [22,23]

$$
f=\frac{2 D_{s} \pi \varepsilon}{\alpha_{s} h^{3} \Delta G_{v o l}} \exp \left(-\frac{\pi \varepsilon^{2}}{k T h \Delta G_{v o l}}\right),
$$

where $D_{s}$ is the interface diffusion coefficient and $\alpha_{s}$ is the average jump distance. If the activation energy were constant, all particles would be expected to begin rounding off at the same temperature. However, figures 5 and 6 show that this is clearly not the case, and the reason for the size dependence can be understood from the form of the driving force. $\Delta \mathrm{G}_{\mathrm{vol}}$ is proportional to $1 / \mathrm{R}$ because it describes the difference in surface energy per volume gained by changing to the equilibrium particle shape ( $\mathrm{R}$ is the particle size). As a first approximation the non-equilibrium, partly faceted shape is assumed to be a cuboctahedron with $\{100\}$ planar facets that approximate the spherical caps. To obtain the maximum driving force the surface energy of this cuboctahedron is compared to that of a sphere of equal volume. For a regular cuboctahedron, geometrical considerations show that this difference corresponds to about $9 \%$ of the specific surface energy of a sphere. The driving force for rounding is thus

$\Delta \mathrm{G}_{\mathrm{vol}}=0.09 \gamma_{P b / A l} S_{\text {sphere }} / V_{\text {sphere }}=\frac{0.27}{R} \gamma_{P b / A l}$.

It can be seen that the driving force $\Delta \mathrm{G}_{\mathrm{vol}}$ decreases with increasing particle size, corresponding to an increase of the energy barrier $\Delta \mathrm{G}^{*}$ for particle rounding. Combining equations 2 and 4, we see that the activation energy is directly proportional to size: 


$$
\Delta G^{*}=\frac{\pi \varepsilon^{2}}{h 0.27 \gamma} R
$$

This size dependence explains our observation, shown in Figure 2, that at a given temperature, larger particles are more anisotropic than smaller particles. Typically, all solid particles have a large aspect ratio, and upon melting, the driving force for rounding is smaller for the larger particles. Hence the larger inclusions remain more anisotropic.

To understand the dependence on temperature, we consider equ. 5. For any inclusion to exhibit observable rounding, the exponential $\Delta \mathrm{G}^{*} / \mathrm{kT}$ must reach a critical value $\mathrm{C}$. Hence,

$$
T_{r}=\frac{\pi \varepsilon^{2}}{C k h 0.27 \gamma} R=D^{2} \varepsilon^{2} R
$$

where $\mathrm{D}$ is a constant. This shows that larger inclusions will round off at higher temperatures. For constant step energy $\varepsilon$, this dependence of rounding temperature on size is linear. Experimental observations of the rounding temperature $\mathrm{T}_{\mathrm{r}}$ as a function of size show that $T_{r}$ indeed increases with size. However, as seen in Figure 6 , the dependence is clearly non-linear, indicating that the step energy is not constant. To highlight the dependence of $\varepsilon$ on temperature, we rewrite equ. 6 as

$$
D \varepsilon=\sqrt{T_{r} / R}
$$

and plot $\sqrt{T_{r} / R}$ as a function of T. As seen in Figure 8, there is a clear decrease of $\varepsilon$ with temperature. Using a linear fit and extrapolating, we find that the step energy vanishes at about $600^{\circ} \mathrm{C}$. A similar behavior is obtained directly from Figure 6 where it is apparent that the rounding temperature $T_{r}$ levels off with increasing size and seems to approach a limiting value (see inset dashed line). This behavior indicates an interface roughening transition, corresponding to the disappearance of the step energy. Above this temperature, interfaces are atomically rough and can advance without the need for ledge 
nucleation. Although the critical temperature derived from these kinetic observations is not very accurate, the observation of interface roughening in this system is significant and unambiguous.

\section{Local Equilibrium}

Having established that despite high atomic mobility the approach to equilibrium can be kinetically limited by the need for ledge nucleation, we now accept the kinetic limitation of the $\{111\}$ facets as a fact and attempt to evaluate the true anisotropy of interfacial energy from measurements of the local equilibrium shape. The observations show that after melting, all interface orientations except the $\{111\}$ facets are rough and can round off easily so as to adopt a shape of minimum interface energy. Meanwhile the $\{111\}$ facets are immobile, and may be considered rigid boundaries that contain the liquid within a limiting octahedron. Thus, upon heating, a solid particle will melt and round off in all orientations except the $\{111\}$ facets. The liquid inclusion will then behave approximately like a liquid in an octahedral crevice.

A schematic comparison of typical shapes is shown in Figure 9. The fully faceted shape of a solid inclusion (a) rounds off at the $\{100\}$ facets upon melting (b). The mass transport necessary for this shape change is highlighted by showing the solid particle shape as a dashed outline in (b). This rounding occurs very rapidly (within a fraction of a second) and the associated matrix volume strain is spontaneously accommodated by thermal vacancies. If all interface orientations were equally mobile and isotropic in energy, the particle would adopt a spherical shape. If the $\{111\}$ orientations were mobile but lower in energy than all other orientations, the equilibrium shape would be a sphere truncated by $\{111\}$ facets (see c). However, we have established that $\{111\}$ facets below their roughening transition temperature can be immobile. The $\{111\}$ facets thus constitute an effective octahedral cage confining the liquid $\mathrm{Pb}$. As a result, a constrained 
or local equilibrium is established in which all interface orientations except $\{111\}$ move toward local equilibrium, forming spherical caps that join the $\{111\}$ facets at a characteristic contact angle $\theta$. This characteristic angle indicates a range of orientations that are forbidden on the equilibrium shape (c). The same range of forbidden angles is established locally at the junction of the spherical caps with the facets (d) if kinetic limitations prevent the particle from reaching its global equilibrium shape. Comparing (c) and (d) it is clear that the equilibrium shape (c) and the kinetically limited shape (d) have very different aspect ratios, but identical contact angles between the spherical and flat segments of the interface.

We thus devised a method to measure this contact angle from digitized micrographs using a 7-parameter least squares fit. A complete description of the method is given elsewhere [24]. From these fits, it was possible to calculate the non-equilibrium aspect ratio, the average contact angle $\theta$ and the corresponding equilibrium aspect ratio $\mathrm{c} / \mathrm{a}=1 / \cos \theta$ for each particle, regardless of size or overall shape.

The results of these measurements are shown in Figure 10. The strong size dependence of the measured aspect ratio shown earlier in Figure $2 \mathrm{~b}$ disappears when the equilibrium aspect ratio is calculated from the local equilibrium measurements. This is illustrated directly in Figure 10a for isothermal measurements at $427^{\circ} \mathrm{C}$. Although the local equilibrium shape (solid dots) still shows a fair amount of scatter, the size dependence has vanished and the average anisotropy at this temperature is about $1.8 \%$, much smaller than that reported previously [1]. Figure $10 \mathrm{~b}$ plots the temperature dependence of the equilibrium shape determined from these measurements. From a comparison of the data during heating (open squares) and cooling (solid dots) it is clear that there is no shape hysteresis. It is also apparent that there is a monotonic decrease in aspect ratio with temperature, both, during heating and cooling. A linear fit to both sets of data 
extrapolates to an aspect ratio of 1 (isotropic energy) at $550^{\circ} \mathrm{C}$, indicating the disappearance of the $\{111\}$ facets at their roughening transition temperature.

The maximum anisotropy of about $2.0 \%$ for the equilibrium shape is found from the data in Figure 10 at $350^{\circ} \mathrm{C}$. It is well-known that the facet width w on the equilibrium shape is a direct measure of the step energy $\varepsilon$ [25]. Since a vicinal surface can be described as a series of steps and terraces on the singular surface, its energy $\gamma$ can be written as [26]

$$
\gamma=\gamma_{0}+|\alpha| \varepsilon / h+\ldots
$$

where $h$ is the step height, $\gamma_{0}$ the energy of the singular surface, and $\alpha$ is the inclination angle of the vicinal surface, and higher order terms that describe step-step interactions have been neglected. The step energy is then simply the angular derivative of the vicinal surface energy,

$$
\left.\frac{\partial \gamma}{\partial \alpha}\right|_{\alpha=0}=\frac{\varepsilon}{h}
$$

Following Herring [27], we can write the minimum slope of the $\gamma$-plot at a facet as a segment of a circle that intersects the origin, the center and the edge of the facet:

$$
\gamma(\alpha)=c \cos (\theta-\alpha)
$$

and thus its derivative at the cusp $(\alpha=0)$ in the $\gamma$-plot is

$$
\left.\frac{\partial \gamma}{\partial \alpha}\right|_{\alpha=0}=c \sin \theta
$$

From Figure $9 \mathrm{c}$ it can be seen that $c \sin \theta=\mathrm{w} / 2=\mathrm{c} \sqrt{1-\frac{a^{2}}{c^{2}}}$. Combining equations 9 and 11 , and equating $\mathrm{c}$ with $\gamma_{\mathrm{Al} / \mathrm{Pb}}$, the isotropic interface energy between solid $\mathrm{Al}$ and liquid $\mathrm{Pb}$, we obtain

$$
\frac{\varepsilon}{\gamma_{\mathrm{Al} / \mathrm{Pb}}}=\mathrm{h} \sqrt{1-\frac{a^{2}}{c^{2}}}
$$


For an anisotropy c/a=1.02 obtained from Figure $10, \mathrm{~h}=0.23 \mathrm{~nm}$ (the spacing of $\{111\}$

planes in $\mathrm{Al}$ ), and using McLean's value of $\gamma_{\mathrm{Al} / \mathrm{Pb}}=0.419 \mathrm{~J} / \mathrm{m}^{2}$ for the interface energy at $350^{\circ} \mathrm{C}$ [7], we calculate a step energy $\varepsilon=1.9 \cdot 10^{-11} \mathrm{~J} / \mathrm{m}$.

\section{DISCUSSION}

This work has shown that despite their small size and the rapid accommodation of shape changes during melting, $\mathrm{Pb}$ inclusions, once liquid, can be extremely slow to reach equilibrium. This paradox is illustrated in Figure 4, which shows that even for particles only $20 \mathrm{~nm}$ in size two different shapes can remain stable for at least $9 \mathrm{~h}$ at $450^{\circ} \mathrm{C}$. A highly anisotropic shape with large facets is stable during heating, and a much more rounded shape is stable during cooling after a temperature excursion to $500^{\circ} \mathrm{C}$. In view of the rapid mobility at much lower temperatures, the explanation of these effects in terms of a kinetic limitation may seem unlikely. However, the analysis shows that these two facts are not incompatible. In a faceted particle, the approach to equilibrium is asymmetric because a convex interface can grow only by nucleating ledges on the facets, but can shrink simply by atomic attachment to existing ledges. For Au particles this asymmetry has been shown explicitly by approaching the equilibrium shape of a truncated sphere from different initial non-equilibrium shapes [28]. Evolution toward equilibrium from a tabular shape was much slower than from a spherical shape because of the need for ledge nucleation.

The requirement for the nucleation of ledges to allow inclusions to approach equilibrium is illustrated schematically in Figure 7. Although the step energy is not large, the driving force (the gain in interface energy per volume upon equilibration) is quite small and decreases with increasing particle size. As a result, the nucleation barrier is high and increases with size. This leads to the observed behavior of size-dependent rounding. Only when the thermal energy becomes sufficient to overcome the nucleation barrier for a 
given particle size, can such inclusions get closer to equilibrium. Notice, however, that as the shape approaches equilibrium, the driving force diminishes further. Thus, complete rounding can occur only when the step energy disappears completely. From kinetic observations of the temperature at which inclusions of a given size begin to round off, it was possible to estimate a roughening temperature of $\mathrm{T}_{\text {rough }}=600^{\circ} \mathrm{C}$ where the step energy $\varepsilon$ vanishes.

Using an even simpler model, analogous to that of Willertz and Shewmon who analyzed the diffusion of small $\mathrm{He}$ bubbles in $\mathrm{Au}$ and $\mathrm{Cu}$ [22], one could neglect the driving force for shape change and assume that a new island forms only if the nucleus covers the entire facet. For such a nucleus, the step energy becomes subsumed in the energy of the edge encircling the facet. This model leads to a critical nucleation barrier $\Delta G^{*}=\pi \varepsilon r$ (equivalent to equation 5) and a temperature dependence $T_{r}=C \varepsilon r$ (equivalent to equation 6). Using this form to fit the kinetic observations, we obtain a linear fit of similar statistical quality, with a roughening temperature of $\mathrm{T}_{\text {rough }}=510^{\circ} \mathrm{C}$. Notice that neither procedure can be considered accurate because we are simply using a linear extrapolation of kinetic data with a fairly large degree of scatter. The main conclusion that can be drawn from this analysis is that $\varepsilon$ decreases with temperature and that there exists a roughening transition for the $\{111\}$ facets of the interface between solid $\mathrm{Al}$ and liquid $\mathrm{Pb}$.

A better estimate of the roughening temperature was obtained from measurements of local equilibrium. Making use of the observation that all interface orientations except $\{111\}$ facets are free to move, the junction between the spherical caps and the octahedral facets was utilized to estimate the equilibrium shape. The fact that these results were essentially identical during heating and cooling, and there was no noticeable size dependence proves that we are indeed observing equilibrium. As seen in Figure 10, the equilibrium shape displays a gradual but significant slope. A similar slope is observed in 
the aspect ratios shown in Figure 5, during the cooling cycle, although there is a systematic difference in the magnitude of the aspect ratio. This is probably due to the fact that heating to $500^{\circ} \mathrm{C}$ was insufficient for the particles to round off completely. Figure 10 shows a critical roughening temperature of $550^{\circ} \mathrm{C}$, about halfway between the two estimates from kinetic data.

Evidence for roughening transitions in metals has been reported previously for the (0001) interface of solid $\mathrm{Zn}$ in contact with four different binary alloy melts [29] by measuring the shapes of liquid alloy inclusions $10-30 \mu \mathrm{m}$ in size after equilibration in a Zn-rich matrix. It was found that for monotectic systems, the roughening transition coincided with the monotectic temperature. Although $\mathrm{Al}-\mathrm{Pb}$ also forms a monotectic system, the roughening transition temperature of $550^{\circ} \mathrm{C}$ obtained here is well below the monotectic temperature of $659^{\circ} \mathrm{C}$. The conclusion that the $\{111\}$ interface between liquid $\mathrm{Pb}$ and solid Al is atomically rough well below the monotectic temperature is supported by the observation of spheroidization of micron-sized $\mathrm{Pb}$ inclusions at about $600^{\circ} \mathrm{C}[7,8]$, and from the observation that the $\{100\} \mathrm{Pb}-\mathrm{Al}$ interface undergoes roughening even before the inclusion melts [15].

The data in Figure 10 also allowed us to estimate the maximum anisotropy of the interface energy of $2.4 \%$ at the melting point of $\mathrm{Pb}\left(327^{\circ} \mathrm{C}\right)$. This is much smaller than previous reports of $11 \%[6]$ or $14 \%[1,11]$ which were most likely due to kinetic limitations. The observation in those investigations of a transformation to a nearspherical shape was probably the rounding temperature for this particle size (but still below the roughening temperature). The lower value for the interface anisotropy found in the present work is in agreement with the report of Camel et al. [10].

Although Figure 10 shows a clear temperature dependence of the equilibrium shape, the accuracy of the data is insufficient to determine the form of this relationship, and the 
linear fit employed is empirical. However, using a regular solution model for the solid/solid interfacial energy of GP zones in Al-Ag alloys, Alexander et al. [30] predicted a linear temperature dependence of the anisotropy, and a roughening temperature of 250$440^{\circ} \mathrm{C}$ (depending on the model). By comparison, the experimental observations extrapolated to about $500^{\circ} \mathrm{C}$. However, the accuracy of these data was also insufficient to distinguish between different models. More accurate measurements on much larger $\mathrm{Pb}$ particles in equilibrium with their vapor showed that the temperature dependence of the surface energy anisotropy was not linear [31].

Similarly, our shape measurements are not sufficiently accurate to allow us to determine the precise form of the curved sections of the equilibrium shape where it meets the flat facets. This curvature is an important parameter to measure in order to distinguish between different models of surface steps and their interactions [32]. However, even on larger particles imaged in a scanning electron microscope, such measurements are notoriously difficult to make $[33,34]$. On the other hand, using a scanning tunneling microscope, Arenhold et al. [35] were recently able to accurately measure facets on free $\mathrm{Pb}$ particles about $2 \mu \mathrm{m}$ in size. The relative facet size and shape were then used to obtain values for the step energy as a function of step direction. From these measurements it was also possible to derive step stiffness and interaction energies.

For nanoscale inclusions imaged in a transmission electron microscope, shape measurements are complicated by the fact that the edge contrast is determined by the particle's projected thickness. This makes it hard to determine the precise boundary of a particle that is curved in the beam direction, whereas flat facets that are parallel to the beam are easily located. Future measurements on larger particles embedded in thicker foils, fully equilibrated at temperatures above $T_{\text {rough }}$ should be able to give significantly higher accuracy, especially if weak beam imaging conditions are employed to measure thickness contours. 


\section{SUMMARY}

In-situ electron microscopy observations of the shape of liquid $\mathrm{Pb}$ inclusions in a solid $\mathrm{Al}$ matrix were made at temperatures between 300 and $500^{\circ} \mathrm{C}$. Careful measurement of inclusion shapes revealed a clear hysteresis in shape during heating and cooling. All particles were found to round off at a critical temperature $T_{r}$ which increased non-linearly with inclusion size. This behavior was explained by the need for ledge nucleation during shape equilibration of faceted particles. Despite the kinetic limitation the anisotropy of the interface energy could be derived from measurements of local equilibrium. This anisotropy was $2 \%$, significantly smaller than values reported previously. From the facet width, the step energy at $350^{\circ} \mathrm{C}$ was found to be $\varepsilon=1.9 \cdot 10^{-11} \mathrm{~J} / \mathrm{m}$ and decreased with increasing temperature. Extrapolating from a linear fit of the data, the step energy was predicted to vanish at $550^{\circ} \mathrm{C}$, corresponding to the roughening transition temperature of the $\{111\}$ interface between liquid $\mathrm{Pb}$ and solid $\mathrm{Al}$.

\section{ACKNOWLEDGMENTS}

We are grateful to J.W. Cahn for suggesting the kinetic limitation, to P. Wynblatt, D. Chatain and and S. Hagège for helpful discussions, and to P. Ochin for supplying the melt-spun alloys. This work is supported by the Director, Office of Energy Research, Office of Basic Energy Sciences, Materials Sciences Division of the U.S. Department of Energy under Contract No. DE-ACO3-76SFOOO98 and the Danish Natural Science Research Council. 


\section{FIGURE CAPTIONS}

Figure 1: $\quad$ Phase diagram of Al-Pb illustrating miscibility gap in the liquid state. The solubility of $\mathrm{Pb}$ in solid $\mathrm{Al}$ is insignificant up to the monotectic temperature. The solubility of $\mathrm{Al}$ in solid $\mathrm{Pb}$ is negligible and in liquid $\mathrm{Pb}$ ranges from 0.1 to $2 \%$.

Figure 2: $\quad$ Dependence of inclusion shape on size. a) Array of liquid $\mathrm{Pb}$ particles at $\mathrm{T}=423^{\circ} \mathrm{C}\left(96^{\circ}\right.$ above the bulk melting point), showing that the degree of faceting depends on particle size. The smallest inclusion (arrowed) remains solid. b) Plot of aspect ratio versus size for a constant temperature of $427^{\circ} \mathrm{C}$.

Figure 3: $\quad$ Shape evolution of a single inclusion through a complete temperature cycle, showing different shapes at the same temperature during heating (arrow up) and cooling (arrow down).

Figure 4: Thermal stability of inclusion shape at $450^{\circ} \mathrm{C}$ during the heating and cooling cycles. In a) and b), a group of particles is seen after heating directly to $450^{\circ} \mathrm{C}$ during heating, and after holding at this temperature for $9 \mathrm{~h}$ with no noticeable change in shape. In c) and d) particles of similar size are shown after a brief temperature excursion to $527^{\circ} \mathrm{C}$ (above their rounding temperature), immediately followed by cooling to $450^{\circ} \mathrm{C}$ and after holding for $9 \mathrm{~h}$ at this temperature (micrographs courtesy A. Værnholt Olesen [18]).

Figure 5: Aspect ratio as a function of temperature, illustrating shape hysteresis for three size groups, 50-75nm (averaged over 5 measurements), 75-100nm (averaged over 6 measurements) and 100-150nm (averaged over 6 measurements). Onset of rounding during the heating cycle occurs at lower temperature for smaller sizes. Shape remains steady and independent of size during cooling.

Figure 6: Rounding temperature $\mathrm{T}_{\mathrm{r}}$ versus particle size showing steep increase in small size regime and more gradual increase at larger sizes. 
Figure 7: $\quad$ Superposition of particle shapes during heating at $367^{\circ} \mathrm{C}$ (faceted outline) and $505^{\circ} \mathrm{C}(\mathrm{a})$, illustrating that mass redistribution during shape change involves outward motion beyond the faceted interfaces (b) by nucleation and propagation of ledges.

Figure 8: $\quad$ Step energy $\varepsilon$ as a function of temperature showing linear least squares fit, extrapolated to $\varepsilon=0$ at $600^{\circ} \mathrm{C}$.

Figure 9: $\quad$ Schematic illustration of different inclusion shapes. Before melting, solid inclusions are fully faceted cuboctahedra (a). Upon melting, all orientations except $\{111\}$ round off to form spherical caps that replace the $\{100\}$ facets. This shape change is indicated by solid and dotted lines, respectively in (b). In the equilibrium shape (c), the spherical caps join the $\{111\}$ facets at an angle $\theta$, indicating forbidden orientations in the range of $\pm \theta$ from the facet. The isotropic part of the interface energy is $c$, the facet energy $a$ and the facet width is $w$. The kinetically limited shape (d) maintains the same local geometry as the equilibrium shape, with a spherical cap joining the $\{111\}$ at an angle $\theta$ identical to the angle $\theta$ on the equilibrium shape in (c).

Figure 10: Comparison between and local and global measurements (a) illustrating that local equilibrium is independent of size. (b) Equilibrium aspect ratio $c / a=1 / \cos \theta$ as a function of temperature measured from the forbidden angle $\theta$ with separate linear fits to the data measured during heating (open symbols) and cooling (solid symbols). Although the shapes during heating exhibit more scatter, both sets of data extrapolate to the same roughening temperature of $550^{\circ} \mathrm{C}$. The anisotropy at $350^{\circ} \mathrm{C}$ is about $2 \%$. 


\section{REFERENCES}

1. Moore, K.I., Chattopadhyay, K. and Cantor, B., Proc. Roy. Soc. Lond., 1987, A414, 499

2. Herring, C. in "Structure and Properties of Solid Surfaces", Gomer, R. and Smith, C.S. Eds., 1953, University of Chicago Press, Chicago, , pp. 5-81

3. Dahmen, U., Xiao, S.Q., Paciornik, S., Johnson, E. and Johansen, A., Phys. Rev. Lett., 1997, 78, 471

4. Johnson, E., Johansen, A., Dahmen, U., Chen, S.-J. and Fujii, T., Mat. Sci. Forum, $1999,294,115$

5. Johnson, E., Johansen, A., Hinderberger, S., Xiao, S.Q. and Dahmen, U., Interface Science, 1996, 3, 279

6. Thackery, P.A. and Nelson, R.S., Phil. Mag., 1969, 19, 169

7. McLean, M., Phil Mag., 1973, 27, 1253

8. McLean, M.and Loveday, M.S., J. Mat. Sci., 1974, 9, 1104

9. McCormick, M.A., Evans, E.B. and Erb, U., Phil. Mag. Lett., 1986, 53, L27

10. Camel, D., Simon, J.P. and Eustasthopoulos, N., Scripta Met., 1980, 14, 1101

11. Moore, K.I., Zhang, D.L. and Cantor, B., Acta Met. Mat., 1990, 38, 1327

12. Johnson, E., Gråbæk, L., Bohr, J., Johansen, A., Sarholt-Kristensen, L. and Andersen, H.H., MRS Proc., 1990, 157, 247

13. Gråbæk, L., Bohr, J., Johnson, E., Johansen, A., Sarholt-Kristensen, L. and Andersen, H.H., Phys. Rev. Lett., 1990, 64, 934

14. Zhang, D.L. and Cantor, B., Acta Met. Mat., 1991, 39, 1595 
15. Gråbæk, L., Bohr, J., Andersen, H.H., Johansen, A., Johnson, E., SarholtKristensen, L. and Robinson, I.K., Phys. Rev., 1992, B 45, 2628

16. Zhang, D.L. and Cantor, B., Scr. Met., 1990, 24, 751

17. Kim, W.T. and Cantor, B., Acta Met. Mat., 1992, 40, 3339

18. Værnholt Olesen, A., MS thesis, 1998, University of Copenhagen

19. Kjeldgaard, L., Ph.D. thesis, 1999, University of Copenhagen

20. Gabrisch, H., Kjeldgaard, L., Vaernholt Olesen, A., Johnson, E. and Dahmen, U., Proc. MRS, Fall 1999, in press

21. Gabrisch, H., Cong, G., Parvin, B., Kjeldgaard, L., Johnson, E. and Dahmen, U., Micr. and Microanalsysis, 2000, 6, suppl. 2, 1062

22. Willertz, L.E. and Shewmon, P.G., Met. Trans., 1970, 1, 2217

23. Goodhew, P.J. and Smith, D.A., Scr. Met., 1982, 16, 69

24. Gabrisch, H. et al. to be published

25. Wortis, M. in "Chemistry and Physics of Solid Surfaces", Vanselow, R. and Howe, R. (eds), 1988, Springer, New York, Vol. 7, p.367

26. Zangwill, A., Physics at Surfaces, 1991, Cambridge University Press

27. Herring, C., Phys. Rev., 1951, 82, 87

28. Métois, J.J. and Heyraud, J.C., J. Cryst. Growth, 1982, 57, 487

29. Passerone, A. and Eustasthopoulos, N., Acta Met., 1982, 30, 1349

30. Alexander, K.B., LeGoues, F.K., Aaronson, H.I. and Laughlin, D.E., Acta Met., 1984, 32, 2241

31. Heyraud, J.C. and Métois, J.J., Surf. Sci., 1983, 128, 334 
32. Jeong, H.C. and Williams, E.D., Surf. Sci. Rep., 1999, 34, 171

33. Métois, J.J. and Heyraud, J.C., Surf. Sci., 1987, 180, 647

34. Rottman, C., Wortis, M., Heyraud, J.C. and Métois, J.J., Phys. Rev. Lett., 1984, 52, 1009

35. Arenhold, K., Surnev, S., Bonzel, H.P. and Wynblatt, P., Surf. Sci., 1999, 424, 271 